\title{
Efficacy and safety of apatinib combined with transarterial chemoembolization (TACE) in treating patients with recurrent hepatocellular carcinoma
}

\author{
Huiying Gu\#, Jing Li", Nan You", Ke Wu, Zheng Wang, Liang Wang, Yinan Zhu, Qinqin Liu, \\ Xuehui Peng, Lu Zheng^ \\ Department of Hepatobiliary Surgery, Xinqiao Hospital, Army Medical University, Chongqing, China \\ \#These authors contributed equally to this work. \\ Correspondence to: Lu Zheng, MD, PhD. Department of Hepatobiliary Surgery, Xinqiao Hospital, Army Medical University, 183 Xinqiao Street, \\ Shapingba District, Chongqing 400037, China. Email: xqyyzl1@163.com.
}

Background: Apatinib, a vascular endothelial growth factor receptor 2 (VEGFR-2) inhibitor, has shown promising therapeutic effect for hepatocellular carcinoma (HCC). This prospective clinical study was implemented to evaluate the efficacy and safety of apatinib combined with transarterial chemoembolization (TACE) versus TACE alone in treating patients with recurrent HCC after hepatectomy.

Methods: Eligible patients with postoperative recurrent HCC from January 2018 to January 2020 were enrolled at the Xinqiao Hospital of Army Medical University. Patients were randomized 1:1 into TACE plus apatinib group or TACE-alone group. The clinical information of patients was collected, and the patients were followed up until untreatable progression or the end of the study. Adverse events (AEs), overall survival (OS) and progression-free survival (PFS) between the two groups were evaluated. In addition, the objective response rate (ORR) and the disease control rate (DCR) were determined according to the modified Response Evaluation Criteria In Solid Tumors (mRECIST). Among those indexes, PFS was the primary endpoint.

Results: This study enrolled 80 patients with recurrent HCC, and the demographics and primary tumor characteristics were balanced between the two groups. However, TACE plus apatinib treatment could significantly improve the median PFS of patients when compared with the TACE-alone group (17.2 vs. 12.5 months, $\mathrm{P}=0.041$ ). The 1- and 2-year overall survival (OS) rates showed a tendency of improving in the TACE plus apatinib group, but not significantly (95.0\% vs. $85.0 \%$, and $90.0 \%$ vs. $75.0 \%$; both P>0.05). Furthermore, the TACE plus apatinib treatment did significantly increase the short-term ORR and DCR when compared with the TACE-alone group (all $\mathrm{P}<0.05$ ). And no unexpected toxicity or procedure-related mortality was occurred during this study.

Conclusions: The combination treatment of apatinib and TACE might be safe and of potential benefit on patients with intrahepatic recurrent HCC.

Keywords: Hepatocellular carcinoma (HCC); transarterial chemoembolization (TACE); apatinib

Submitted Oct 15, 2020. Accepted for publication Nov 26, 2020.

doi: $10.21037 / \mathrm{atm}-20-7244$

View this article at: http://dx.doi.org/10.21037/atm-20-7244

^ ORCID: 0000-0001-9198-4380. 


\section{Introduction}

Hepatocellular carcinoma (HCC) is one of the fastest growing malignant diseases in the world $(1,2)$. Despite the efforts made towards achieving a long-term cure, postoperative tumors recur in more than $50 \%$ patients within 5 years (3). This necessitates further treatment by performing re-resection or salvage transplantation $(4,5)$. However, not all patients are amenable to further surgery due to limited preservation of liver function, difficult tumor location, and/or unavailability of liver transplantation.

Transarterial chemoembolization (TACE) has been developed as a readily available treatment option with good tolerance, limited liver toxicity, and demonstrated efficacy for intermediate-stage HCC, even in recurrent patients with borderline liver function (5-7). However, the local hypoxia environment caused by interventional embolization could stimulate tumor angiogenesis and increase the expression of vascular endothelial growth factor (VEGF), which in turn promotes tumor recurrence and metastasis $(8,9)$. Short-term tumor progression has been reported in some patients after TACE treatment, and the overall effect remains unsatisfactory $(8,10)$. Therefore, it is reasonable that the therapy of TACE combined with anti-angiogenic drugs would be conducive to augment the clinical benefit of TACE alone.

Apatinib, a novel oral anti-angiogenic small molecule that blocks VEGFR-2, has been approved as a 3rd line or beyond therapy in treating patients with advanced gastric or gastroesophageal junction adenocarcinoma in China (11). Studies have shown the promising therapeutic potential of apatinib on various types of cancers, including HCC $(12,13)$. Moreover, the strategy of apatinib combined with TACE has been elaborated to be safe and effective on patients with intermediate or advanced HCC (14-18). Nevertheless, there was no study have focused on evaluating the efficacy of TACE plus apatinib in treating patients with postoperative recurrent HCC.

Therefore, this prospective clinical study was conducted to evaluate the efficacy and safety of TACE with apatinib versus TACE alone in treating patients with recurrent HCC.

We present the following article in accordance with the CONSORT reporting checklist (available at http://dx.doi. org/10.21037/atm-20-7244).

\section{Methods}

\section{Participants}

Patients with recurrent HCC defined by clinical, biochemical, and imaging evaluation between January 2018 and January 2020 at the Xinqiao Hospital of Army Medical University, were randomized (1:1) into TACE plus apatinib group or TACE-alone group by using the block randomization method. No patient was allowed to switch groups once randomized. The clinical information of the patients was prospectively collected, and all participants were followed up until the end of the study or untreatable progression, which was defined as untreatable tumor progression, transient deterioration to Child-Pugh C, or appearance of vascular invasion/extra-hepatic metastases. The study was registered on the Chinese Clinical Trial Registry (http://www.chictr.org.cn; no. ChiCTRIPR-17012667). All procedures performed in this study involving human participants were in accordance with the Declaration of Helsinki (as revised in 2013). The protocol was approved by the China Ethics Committee of Registering Clinical Trials (ethics committee approval no. ChiECRCT-20170060). And all the patients provided written informed consent before enrollment.

\section{Inclusion and exclusion criteria}

Eligible patients needed to meet the following criteria: (I) aged 18 years or older; (II) diagnosed with HCC by two imaging modalities or biopsy; (III) intrahepatic recurrence after hepatic resection; (IV) Barcelona Clinic Liver Cancer (BCLC) stage B/C; (V) Child-Pugh class A/ $\mathrm{B}$; (VI) could not tolerate or refuse performing re-resection or salvage transplantation; (VII) adequate organ function; (VIII) an Eastern Cooperative Oncology Group (ECOG) performance status (PS) score of 0-2; and (IX) no vascular invasion or extra-hepatic metastasis. Patients were excluded if they met any of the following criteria: (I) combined use of other anticancer therapy after the surgery; (II) accompanied by other malignancies; (III) severe coagulation disorders; (IV) serious medical comorbidity; and (V) a large amount of ascites or refractory ascites. 


\section{Therapeutic methods}

\section{TACE procedure}

TACE was performed following a previously reported method (14). Briefly, the modified Seldinger technique was used for hepatic artery catheterization. Both superior mesenteric and common hepatic arteriography were performed with a 5 - or 4-F catheter to assess the overall anatomy, tumor burden, and the patency of the portal vein. Next, conventional super-selective catheterization was carried out for the hepatic artery and other feeding arteries. Then, hepatic artery infusion chemotherapy was performed using $50 \mathrm{mg}$ of lobaplatin (Hainan Changan International Pharmaceutical Co., Ltd., Hainan, China). Subsequently, chemoembolization was performed with $50 \mathrm{mg}$ of epirubicin (Shenzhen Wanle Pharmaceutical Co., Ltd., Guangdong, China) mixed with $5-10 \mathrm{~mL}$ of ethiodized oil (Jiangsu Hengrui Medicine Co., Ltd., Jiangsu, China), followed by the application of 8 Spheres microspheres 100-300 $\mu \mathrm{m}$ in diameter (Jiangsu Hengrui Medicine Co., Ltd., Jiangsu, China), until blood flow in the feeding arteries stagnated. The dose of ethiodized oil was determined by the surgeon according to tumor size, blood supply of tumor, and other factors. In order to prevent nausea and vomiting after each TACE procedure, symptomatic treatments were applied to protect the gastric and liver function. Further TACE were performed on an "on-demand" basis as previously reported (19).

\section{Apatinib administration}

Oral apatinib (Jiangsu Hengrui Medicine Co., Ltd., Jiangsu, China) at a dose of $500 \mathrm{mg}$ qd was given to patients in the TACE plus apatinib group beginning the third day after the first TACE treatment and was suspended 3 days before and after the following TACE treatment procedure. Dosage modified to $250 \mathrm{mg}$ qd or temporary interruption were permitted in patients with grade 3-4 adverse events (AEs) or treatment-related intolerance until the adverse symptoms remitted to a grade $\leq 2$. Medication of apatinib was continued until significant disease progression, drug intolerance, consent withdrawal, or patient death.

\section{Clinical and laboratory evaluation}

The entire medical history and related records of each patient were collected. The clinical data included age, gender, ECOG PS and Child-Pugh score, BCLC stage and recurrence time. The laboratory data included $\alpha$-fetoprotein (AFP), and hepatitis B surface antigen (HBsAg) status.
Moreover, imaging data concerning tumor size and tumor number were collected through abdominal contrastenhanced computed tomography (CT) and/or magnetic resonance imaging (MRI).

To assess the tumor response, evaluation by CT/MRI every 4-12 weeks after TACE treatment was administrated according to the modified response evaluation criteria in solid tumors (mRECIST, 2008). In addition, the overall survival (OS, defined as the interval between the first TACE procedure and the time of all-cause death) and PFS (defined as the time from the first TACE procedure to disease progression or all-cause death) were appraised in the two groups, while PFS was the primary endpoint. Toxicities were assessed on the basis of the National Cancer Institute Common Terminology Criteria for Adverse Events (NCI CTCAE) version 4.03, and all serious AEs and their severities were recorded.

\section{Sample size}

The sample size was based on the primary efficacy endpoint, PFS. The expected median PFS for TACE plus apatinib and TACE alone was assumed to be 18 and 10 months, respectively. The allocation ratio was $1: 1$. The required number of patients was 80 with a dropout rate of $10 \%$ to achieve $80 \%$ power at a one-sided $\alpha$ value of 0.15 .

\section{Statistical analysis}

All statistical analyses were conducted using SPSS version 18.0 (SPSS Inc., Chicago, IL, USA) Continuous variables are expressed as mean \pm standard deviation or median (firstthird quartile), and were compared with an independent $t$-test or Mann-Whitney $U$ test. Categorical variables are expressed as percentage (number/total), and were compared with chi square or Fisher's exact test. Survival curves were plotted for both groups using Kaplan-Meier analysis with the log-rank test. All statistical analysis was based on a twotailed hypothesis test and a $\mathrm{P}$ value $<0.05$ was considered statistically significant.

\section{Results}

\section{Clinical characteristics}

Between January 2018 and January 2020, 80 patients with recurrent HCC who met the selection criteria were included in the present study and randomly assigned to TACE plus apatinib $(n=40)$ group or TACE-alone group 
$(\mathrm{n}=40)$. The two groups showed no significant differences in baseline demographic or laboratory characteristics, including age, gender, Child-Pugh classification, ECOG PS, BCLC stage, level of AFP, tumor number, tumor size, hepatic cirrhosis, hepatitis B, and recurrence time (Table 1).

\section{PFS and OS}

The median PFS was significantly improved in the TACE plus apatinib group compared with the TACE-alone group [17.2 months, 95\% confidence interval (CI): 13.6-20.8 vs. 12.5 months, $95 \% \mathrm{CI}: 11.3-13.7$, hazard ratio (HR) $=0.563,95 \%$ CI: $0.336-0.943, \mathrm{P}=0.041]$ (Figure 1). At the end of the follow-up, more patients survived in the TACE plus apatinib group than in the TACE-alone group [90.0\% (36/40) vs. $75.0 \%$ (30/40)], but the median OS has not yet been reached in either group, and the differences were not statistically significant (HR $=0.360,95 \%$ CI: $0.113-1.150$, $\mathrm{P}=0.072$ ) (Figure 2). The 1- and 2-year OS rates in the two groups were $95.0 \%$ vs. $85.0 \%(\mathrm{P}=0.136)$ and $90.0 \%$ vs. $75.0 \%(\mathrm{P}=0.077)$, respectively.

\section{Tumor response}

Changes could be observed before and after TACE procedure in tumors in both groups on abdominal contrastenhanced CT or MRI scans (Figure $3 A, B, C, D, E, F, G, H$ ). At 1 month and 3 months after the treatment, the tumor responses of all patients were evaluated in both groups according to the mRECIST (Table 2). None of the patients achieved complete response (CR). The TACE plus apatinib group had a significantly higher 1-month objective response rate $(\mathrm{ORR})$ and 1 -month disease control rate (DCR) than the TACE group $(75.0 \%$ vs. $50.0 \%, \mathrm{P}=0.021 ; 90.0 \%$ vs. $72.5 \%, \mathrm{P}=0.045$, respectively) (Table 2 ); and the 3 -month ORR and DCR showed similar trends $(55.0 \%$ vs. $32.5 \%$, $\mathrm{P}=0.043$ and $75.0 \%$ vs. $52.5 \%, \mathrm{P}=0.036$, respectively) (Table 2).

\section{AEs}

Two categories of AEs were considered in this study: postembolization syndrome (PES) and apatinib-related AEs. Features of PES typically include fever, abdominal pain, nausea and vomiting, and myelosuppression. No significant difference was found in the incidence of PES between the two groups (all $\mathrm{P}>0.05$ ) (Table 3).

The incidence of apatinib-related AEs was $80.0 \%(32 / 40)$ in the TACE plus apatinib group (Table 3). Treatmentrelated AEs in the TACE plus apatinib group included the following: hand-foot syndrome (16/40, 40.0\%), hypertension $(11 / 40,27.5 \%)$, fatigue $(10 / 40,25.0 \%)$, diarrhea $(9 / 40,22.5 \%)$, oral ulcer $(9 / 40,22.5 \%)$, and proteinuria $(6 / 40,15.0 \%)$. The majority of toxicities were grade 1-2, which is consistent with the known toxicities associated with apatinib. Once the patients developed grade $3 \mathrm{AE}$, the dose of apatinib was reduced from $500 \mathrm{mg}$ qd to $250 \mathrm{mg}$ qd until remission of symptoms, and the patients could then resume apatinib at $500 \mathrm{mg}$ per day

Thus, no patient developed grade 4 toxicity. Nevertheless, the addition of apatinib to TACE significantly increased the incidence of apatinib-related complications compared to treatment with TACE alone (all $\mathrm{P}<0.05$, Table 3). No unexpected toxicity or procedure-related mortality was observed in either group, and all AEs were effectively alleviated after symptomatic treatment.

\section{Discussion}

HCC recurrence is a critical failure for patients treated with surgical resection (20). Various therapies, such as re-resection, salvage liver transplantation, TACE, radiofrequency ablation, radiotherapy, and systemic therapy (sorafenib or FOLFOX4 chemotherapy) have been applied for intrahepatic tumor recurrence after hepatic resection, and comparisons among these treatment modalities have been reported $(4,5,7,21,22)$. However, there is currently no agreement on a standard guideline in clinical practice for intrahepatic recurrent HCC after hepatectomy.

TACE has been used as a common treatment for recurrent HCC when tumors are unresectable or transplantation is infeasible by virtue of its strong cytotoxic and ischemic antitumor effect, minimal invasiveness, and repeatability $(7,23)$. On the other hand, TACE may promote an embolization-induced local hypoxic environment which can in turn cause neovascularization in tumors $(8,9)$. Previous studies also confirmed that the overexpression of an angiogenic factor, VEGF, could promote recurrence and metastasis in remnant peripheral tumor tissues $(24,25)$. Therefore, inhibiting VEGF pathway may be a crucial strategy for improving the long-term benefit of patients treated with TACE.

Sorafenib is an anti-angiogenic agent, which inhibits multi-kinases, inhibiting RAF kinase, VEGFR-2, VEGFR-3, and PDGFR-b tyrosine kinase in particular. It has been the first-line option for use as an oral targeted 
Table 1 Baseline characteristics of the two groups

\begin{tabular}{|c|c|c|c|}
\hline Characteristic & TACE group $(n=40)$ & TACE plus apatinib group $(n=40)$ & $P$ value \\
\hline Gender, n (\%) & & & 0.502 \\
\hline Male & $22(55.0)$ & $19(47.5)$ & \\
\hline Female & $18(45.0)$ & $21(52.5)$ & \\
\hline A & $35(87.5)$ & $33(82.5)$ & \\
\hline B & $5(12.5)$ & 7 (17.5) & \\
\hline ECOG PS, n (\%) & & & 0.576 \\
\hline $0-1$ & $31(77.5)$ & $33(82.5)$ & \\
\hline B & $33(82.5)$ & $36(90.0)$ & \\
\hline $\mathrm{C}$ & $7(17.5)$ & $4(10.0)$ & \\
\hline Level of AFP ( $\mu \mathrm{g} / \mathrm{L}), \mathrm{n}(\%)$ & & & 0.108 \\
\hline$<400$ & $12(30.0)$ & $19(47.5)$ & \\
\hline$\geq 400$ & $28(70.0)$ & $21(52.5)$ & \\
\hline Tumor number, n (\%) & & & 0.370 \\
\hline Single & $17(42.5)$ & $21(52.5)$ & \\
\hline Multiple & $23(57.5)$ & $19(47.5)$ & \\
\hline No & $19(47.5)$ & $22(55.0)$ & \\
\hline Hepatitis B, n (\%) & & & 0.617 \\
\hline Yes & $30(75.0)$ & $28(70.0)$ & \\
\hline No & $10(25.0)$ & $12(30.0)$ & \\
\hline Recurrence time (year), n (\%) & & & 0.256 \\
\hline$<1$ & $26(65.0)$ & $21(52.5)$ & \\
\hline$\geq 1$ & $14(35.0)$ & $19(47.5)$ & \\
\hline
\end{tabular}

TACE, transarterial chemoembolization; ECOG, Eastern Cooperative Oncology Group; PS, performance score; BCLC, Barcelona Clinic Liver Cancer; AFP, $\alpha$-fetoprotein. 


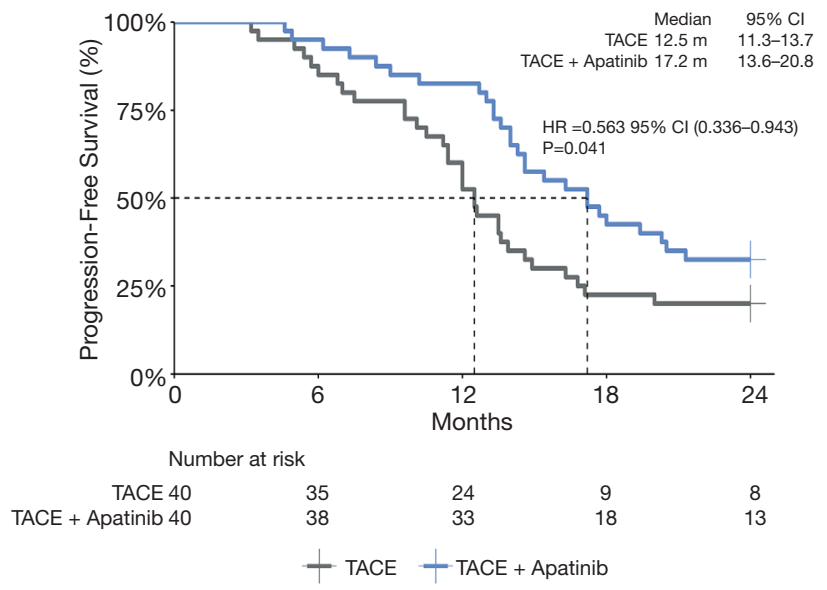

Figure 1 Progression-free survival (PFS) in the transarterial chemoembolization (TACE) plus apatinib and TACE-alone groups. The Kaplan-Meier analysis was used to evaluate PFS in patients. The log-rank test was used to compare treatments. CI, confidence interval; HR, hazard ratio.

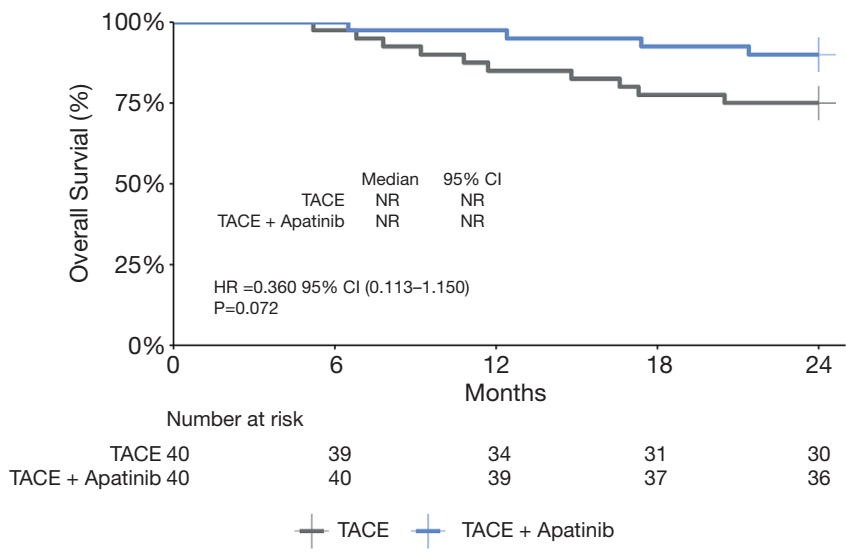

Figure 2 Overall survival (OS) in the transarterial chemoembolization (TACE) plus apatinib and TACE-alone groups. The Kaplan-Meier analysis was used to evaluate OS in patients. The log-rank test was used to compare treatments. CI, confidence interval; NR, not reached; HR, hazard ratio.

agent approved to treat advanced HCC (26). Several analyses have demonstrated that the addition of sorafenib to TACE could produce a synergistic enhancement effect, considerably enhancing OS, time to progression (TTP), and ORR for intermediate- or advanced-stage HCC (27-29). Nevertheless, the data in STORM trial showed there was no significant advantage of sorafenib for HCC following resection or ablation (30). In addition, the remarkable adverse effects and high resistance rate have significantly hampered its use $(31,32)$. Consequently, more effective and less expensive treatments are urgently needed to be explored.

As a highly selective VEGFR-2 blocker, the affinity of apatinib to VEGFR-2 tyrosine kinase is 10 times greater than that of sorafenib (33). Numerous clinical studies have confirmed the efficacy of apatinib for advanced HCC, and further research has corroborated the benefits of TACE plus apatinib for intermediate or advanced HCC (14-16,18). Recently, the RESCUE trial showed that the treatment of apatinib combined with camrelizumab, an anti-programmed death-1 monoclonal antibody, was controllable safe and showed a good curative effect in advanced HCC (34). Therefore, apatinib seems to have a good value in the treatment of HCC.

This prospective randomized controlled study was conducted to investigate the effect of apatinib used concurrently with TACE on recurrent HCC. We found that the apatinib plus TACE treatment resulted in an mPFS benefit of 4.7 months and achieved a higher ORR and DCR when compared with TACE-alone treatment. The results may attribute to the follows: strict inclusion and exclusion criteria, good condition of patients, lack of vascular invasion and extra-hepatic metastasis, and the use of 8Spheres microspheres in TACE treatment ensuring an excellent embolization effect. However, the secondary endpoint of OS was not significantly different between the two groups, which might be partly attributed to the consequence of the limited sample size and the short follow-up time of the study.

Furthermore, the treatments were controllable safe in this study as all serious AEs were effectively alleviated after symptomatic care. The incidence of PES post-embolization showed no significant difference between the two groups. The rate of apatinib-related AEs was higher in the TACE plus apatinib group, with the majority of AEs classified as grade 1-2. No grade $4 \mathrm{AE}$ occurred in this study, which may be explained by the dosage reduction of apatinib once grade $3 \mathrm{AE}$ occurs. Furthermore, no unexpected toxicity or procedure-related mortality was observed.

So far, there is little study regarding on the effect of combination therapy of TACE and anti-angiogenic drugs on patients with recurrence HCC post-hepatectomy. The preliminary results of our study showed that the combination of TACE and apatinib can significantly prolong the mPFS in patients with recurrence HCC, 

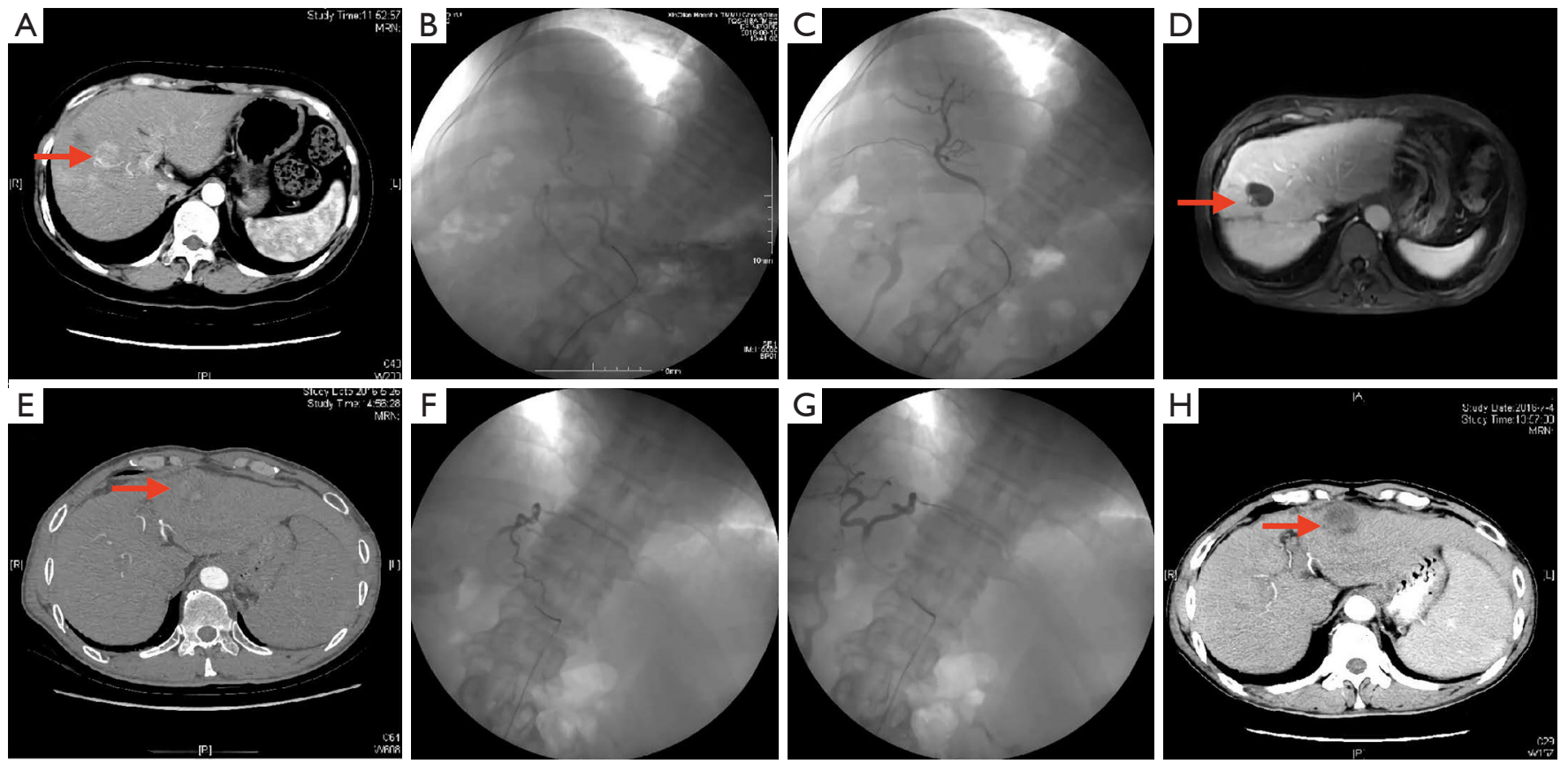

Figure 3 Results of imaging with abdominal contrast-enhanced confidence interval (CT) or magnetic resonance imaging (MRI) in a representative case before transarterial chemoembolization (TACE) and after treatment in both groups and the digital subtraction angiography changes during TACE treatment. Imaging changes before and after TACE in the TACE-alone group (A,D) and TACE plus apatinib group $(\mathrm{E}, \mathrm{H})$. The digital subtraction angiography changes before and after embolization during TACE treatment in the TACEalone group $(\mathrm{B}, \mathrm{C})$ and the TACE plus apatinib group $(\mathrm{F}, \mathrm{G})$. Arrow, the location of tumor.

Table 2 Tumor responses in the two groups

\begin{tabular}{lcc}
\hline Tumor responses & TACE group $(\mathrm{n}=40)$ & TACE plus apatinib group $(\mathrm{n}=40)$ \\
\hline One-month & 0 & 0 \\
CR & $20(50.0 \%)$ & $30(75.0 \%)$ \\
PR & $9(22.5 \%)$ & $6(15.0 \%)$ \\
SD & $11(27.5 \%)$ & $4(10.0 \%)$ \\
PD & $50.0 \%$ & $75.0 \%$ \\
ORR & $72.5 \%$ & $90.0 \%$ \\
DCR & & \\
Three-month & 0 & 0 \\
CR & $13(32.5 \%)$ & $22(55.0 \%)$ \\
PR & $8(20.0 \%)$ & $8(20.0 \%)$ \\
SD & $19(47.5 \%)$ & $10(25.0 \%)$ \\
PD & $32.5 \%$ & $55.0 \%$ \\
ORR & $52.5 \%$ & $75.0 \%$ \\
DCR & 0.045 \\
\hline
\end{tabular}

TACE, transarterial chemoembolization; one-month, the first month after TACE treatment; three-month, the third month after TACE treatment; CR, complete response; PR, partial response; SD, stable disease; PD, progressive disease; ORR, objective response rate, defined as CR+PR; DCR, disease control rate, defined as $C R+P R+S D$. 
Table 3 Adverse events in the two groups

\begin{tabular}{|c|c|c|c|}
\hline Adverse events & TACE group $(n=40)$ & TACE plus apatinib group $(n=40)$ & $P$ value \\
\hline Fever & $10(25.0)$ & $13(32.5)$ & 0.459 \\
\hline Abdominal pain & $14(35.0)$ & $10(25.0)$ & 0.329 \\
\hline Nausea and vomiting & $11(27.5)$ & $14(35.0)$ & 0.469 \\
\hline \multicolumn{4}{|c|}{ Drug-related adverse events, n (\%) } \\
\hline Fatigue & 0 & $10(25.0)$ & $<0.001$ \\
\hline Hand-foot syndrome & 0 & $16(40.0)$ & $<0.001$ \\
\hline Diarrhea & 0 & $9(22.5)$ & 0.001 \\
\hline Oral ulcer & 0 & $9(22.5)$ & 0.001 \\
\hline
\end{tabular}

TACE, transarterial chemoembolization.

suggested that the combination therapy might be safe and of potential benefit in treating patients with intrahepatic recurrent HCC, could be used as a new treatment option for intrahepatic recurrence.

However, our study's findings are limited by the small sample size and a short follow-up time of its design. T10herefore, multicenter, randomized, controlled studies with a larger sample size are needed in the near future to further assess the effects and safety of combining TACE and apatinib in treating recurrent HCC.

\section{Conclusions}

In summary, the results of this study indicated that TACE plus apatinib might be safe and of potential benefit to patients with intrahepatic recurrent HCC after hepatectomy.

\section{Acknowledgments}

Funding: This study was supported by grants from the Scientific and Technological Innovation Projects of Chongqing (no. cstc2017shmsA00011, cstc2017shmsA10005, and cstc2018jscx-mszdX0012).

\section{Footnote}

Reporting Checklist: The authors have completed the
CONSORT reporting checklist. Available at http://dx.doi. org/10.21037/atm-20-7244

Data Sharing Statement: Available at http://dx.doi. org/10.21037/atm-20-7244

Conflicts of Interest: All authors have completed the ICMJE uniform disclosure form (available at http://dx.doi. org/10.21037/atm-20-7244). The authors have no conflicts of interest to declare. The abstract of the study has been orally presented at the ESMO $22^{\text {nd }}$ World Congress on Gastrointestinal Cancer, and has been published in a supplement to the Annals of Oncology. The work described was original research that has not been published in whole previously, and not under consideration for publication elsewhere.

Ethical Statement: The authors are accountable for all aspects of the work in ensuring that questions related to the accuracy or integrity of any part of the work are appropriately investigated and resolved. All procedures performed in this study involving human participants were in accordance with the Declaration of Helsinki (as revised in 2013). The protocol was approved by the China Ethics Committee of Registering Clinical Trials (ethics committee approval no. ChiECRCT-20170060), and all the patients provided written informed consent before enrollment. 
Open Access Statement: This is an Open Access article distributed in accordance with the Creative Commons Attribution-NonCommercial-NoDerivs 4.0 International License (CC BY-NC-ND 4.0), which permits the noncommercial replication and distribution of the article with the strict proviso that no changes or edits are made and the original work is properly cited (including links to both the formal publication through the relevant DOI and the license). See: https://creativecommons.org/licenses/by-nc-nd/4.0/.

\section{References}

1. Bray F, Ferlay J, Soerjomataram I, et al. Global cancer statistics 2018: GLOBOCAN estimates of incidence and mortality worldwide for 36 cancers in 185 countries. CA Cancer J Clin 2018;68:394-424.

2. Kulik L, El-Serag HB. Epidemiology and Management of Hepatocellular Carcinoma. Gastroenterology 2019;156:477-91.e1.

3. Chawla A, Ferrone C. Hepatocellular carcinoma surgical therapy: perspectives on the current limits to resection. Chin Clin Oncol 2018;7:48.

4. Chan ACY, Chan SC, Chok KSH, et al. Treatment strategy for recurrent hepatocellular carcinoma: salvage transplantation, repeated resection, or radiofrequency ablation? Liver Transpl 2013;19:411-9.

5. Zheng S, Xie Q, Cheng J. Salvage liver transplant for hepatocellular carcinoma: rescues and benefits. Transl Gastroenterol Hepatol 2018;3:65.

6. Forner A, Gilabert M, Bruix J, et al. Treatment of intermediate-stage hepatocellular carcinoma. Nat Rev Clin Oncol 2014;11:525-35.

7. Erridge S, Pucher PH, Markar SR, et al. Meta-analysis of determinants of survival following treatment of recurrent hepatocellular carcinoma. Br J Surg 2017;104:1433-42.

8. Pinato DJ, Pai M, Reccia I, et al. Preliminary qualification of a novel, hypoxic-based radiologic signature for transarterial chemoembolization in hepatocellular carcinoma. BMC Cancer 2018;18:211.

9. Petrillo M, Patella F, Pesapane F, et al. Hypoxia and tumor angiogenesis in the era of hepatocellular carcinoma transarterial loco-regional treatments. Future Oncol 2018;14:2957-67.

10. Wang K, Liu G, Li J, et al. Early intrahepatic recurrence of hepatocellular carcinoma after hepatectomy treated with re-hepatectomy, ablation or chemoembolization: a prospective cohort study. Eur J Surg Oncol 2015;41:236-42.
11. Li J, Qin S, Xu J, et al. Randomized, Double-Blind, Placebo-Controlled Phase III Trial of Apatinib in Patients With Chemotherapy-Refractory Advanced or Metastatic Adenocarcinoma of the Stomach or Gastroesophageal Junction. J Clin Oncol 2016;34:1448-54.

12. Scott LJ. Apatinib: A Review in Advanced Gastric Cancer and Other Advanced Cancers. Drugs 2018;78:747-58.

13. Xue JM, Astère M, Zhong MX, et al. Efficacy and safety of apatinib treatment for gastric cancer, hepatocellular carcinoma and non-small cell lung cancer: a meta-analysis. Onco Targets Ther 2018;11:6119-28.

14. Lu W, Jin XL, Yang C, et al. Comparison of efficacy between TACE combined with apatinib and TACE alone in the treatment of intermediate and advanced hepatocellular carcinoma: A single-center randomized controlled trial. Cancer Biol Ther 2017;18:433-8.

15. Fan W, Yuan G, Fan H, et al. Apatinib Combined With Transarterial Chemoembolization in Patients With Hepatocellular Carcinoma and Portal Vein Tumor Thrombus: A Multicenter Retrospective Study. Clin Ther 2019;41:1463-76.

16. Yang Z, Chen G, Cui Y, et al. The safety and efficacy of TACE combined with apatinib on patients with advanced hepatocellular carcinoma: a retrospective study. Cancer Biol Ther 2019;20:321-7.

17. Zhao S, Zhang T, Dou W, et al. A comparison of transcatheter arterial chemoembolization used with and without apatinib for intermediate- to advanced-stage hepatocellular carcinoma: a systematic review and metaanalysis. Ann Transl Med 2020;8:542.

18. Kan X, Liang B, Zhou G, et al. Transarterial Chemoembolization Combined With Apatinib for Advanced Hepatocellular Carcinoma: A Propensity Score Matching Analysis. Front Oncol 2020;10:970.

19. Kim BK, Kim KA, Park JY, et al. Prospective comparison of prognostic values of modified Response Evaluation Criteria in Solid Tumours with European Association for the Study of the Liver criteria in hepatocellular carcinoma following chemoembolisation. Eur J Cancer 2013;49:826-34.

20. Poon RT, Fan ST, Lo CM, et al. Improving survival results after resection of hepatocellular carcinoma: a prospective study of 377 patients over 10 years. Ann Surg 2001;234:63-70.

21. Choi GH, Kim DH, Kang CM, et al. Prognostic factors and optimal treatment strategy for intrahepatic nodular recurrence after curative resection of hepatocellular carcinoma. Ann Surg Oncol 2008;15:618-29. 
22. Tranchart H, Chirica M, Sepulveda A, et al. Long-term outcomes following aggressive management of recurrent hepatocellular carcinoma after upfront liver resection. World J Surg 2012;36:2684-91.

23. Kim SS, Kang TW, Song KD, et al. Radiofrequency ablation and transarterial chemoembolisation as firstline treatment for recurrent hepatocellular carcinoma or isolated intrahepatic recurrent hepatocellular carcinoma in transplanted livers. Clin Radiol 2017;72:141-9.

24. Li Z, Xue TQ, Chen XY. Predictive values of serum VEGF and CRP levels combined with contrast enhanced MRI in hepatocellular carcinoma patients after TACE. Am J Cancer Res 2016;6:2375-85.

25. Wang B, Xu H, Gao ZQ, et al. Increased expression of vascular endothelial growth factor in hepatocellular carcinoma after transcatheter arterial chemoembolization. Acta Radiol 2008;49:523-9.

26. Llovet JM, Ricci S, Mazzaferro V, et al. Sorafenib in advanced hepatocellular carcinoma. $\mathrm{N}$ Engl J Med 2008;359:378-90.

27. Lencioni R, Llovet JM, Han G, et al. Sorafenib or placebo plus TACE with doxorubicin-eluting beads for intermediate stage HCC: The SPACE trial. J Hepatol 2016;64:1090-8.

28. Meyer T, Fox R, Ma YT, et al. Sorafenib in combination with transarterial chemoembolisation in patients with unresectable hepatocellular carcinoma (TACE 2): a randomised placebo-controlled, double-blind, phase 3

Cite this article as: $\mathrm{Gu} \mathrm{H}$, Li J, You N, Wu K, Wang Z, Wang L, Zhu Y, Liu Q, Peng X, Zheng L. Efficacy and safety of apatinib combined with transarterial chemoembolization (TACE) in treating patients with recurrent hepatocellular carcinoma. Ann Transl Med 2020;8(24):1677. doi: 10.21037/atm20-7244 trial. Lancet Gastroenterol Hepatol 2017;2:565-75.

29. Kudo M, Ueshima K, Ikeda M, et al. Randomised, multicentre prospective trial of transarterial chemoembolisation (TACE) plus sorafenib as compared with TACE alone in patients with hepatocellular carcinoma: TACTICS trial. Gut 2020;69:1492-501.

30. Bruix J, Takayama T, Mazzaferro V, et al. Adjuvant sorafenib for hepatocellular carcinoma after resection or ablation (STORM): a phase 3, randomised, double-blind, placebo-controlled trial. Lancet Oncol 2015;16:1344-54.

31. Niu L, Liu L, Yang S, et al. New insights into sorafenib resistance in hepatocellular carcinoma: Responsible mechanisms and promising strategies. Biochim Biophys Acta Rev Cancer 2017;1868:564-70.

32. Zhu YJ, Zheng B, Wang HY, et al. New knowledge of the mechanisms of sorafenib resistance in liver cancer. Acta Pharmacol Sin 2017;38:614-22.

33. Tian S, Quan H, Xie C, et al. YN968D1 is a novel and selective inhibitor of vascular endothelial growth factor receptor-2 tyrosine kinase with potent activity in vitro and in vivo. Cancer Sci 2011;102:1374-80.

34. Xu J, Shen J, Gu S, et al. Camrelizumab in combination with apatinib in patients with advanced hepatocellular carcinoma (RESCUE): a non-randomized, open-label, phase 2 trial. Clin Cancer Res 2020. [Epub ahead of print]. doi: 10.1158/1078-0432.CCR-20-2571.

(English Language Editor: J. Gray) 